\title{
Quantum Jumps: Schrödinger's Objections and the Contemporary Points of View
}

\author{
Sotirios Sakkopoulos \\ Department of Physics, University of Patras, 26500 Patras, Greece \\ Email: ssakkopoulos450@gmail.com
}

\author{
Article History \\ Received: October 24, 2021 \\ Revised: December 10, 2021 \\ Accepted: December 13, 2021 \\ Published: December 17, 2021
}

\begin{abstract}
In 1913, when Bohr's atom allowed electron jumps between discrete orbits, it was impossible to experiment on single atoms and study individually these jumps. Many people considered them not real, but something artificial for the explanation of the linear spectra of atoms. Especially Schrödinger rejected the image of negatively charged electrons orbiting around the nucleus and in particular their jumps from orbit to orbit. Finally, in 1986 single atoms (ions) laser cooled and confined in an electromagnetic trap gave the possibility to investigate experimentally electron jumps to and fro a metastable state. Recently, physicists experimenting on a macroscopic superconducting circuit with discrete quantum states analogous to a natural atom were able to halt and reverse quantum jumps mid-flight revealing very interesting details of them.
\end{abstract}

Keywords: Quantum jumps in single atoms; Artificial atom; Pause and reverse of jumps; Quantum Zeno effect.

\section{Introduction}

In 1913 Niels Bohr proposed a model of atom in which electrons orbiting all-round the nucleus could jump up and down discrete orbits with simultaneous absorption or emission of photons. This produced linear spectra originated from a great number of atoms masking the effect from any single of them. At that time nobody could investigate experimentally individual quantum electron jumps and probe the physics of their movement. Many people thought that these jumps were just artifacts convenient to explain the linear spectra.

In the fall of 1926 Schrödinger visited Bohr in Copenhagen and the latter tried with insistency to persuade him, in vein, that his model was correct. The image of charged particles like electrons orbiting without radiating electromagnetic waves as Maxwell's theory predicted was unacceptable to Schrödinger. Especially, he opposed quantum jumps between orbits wondering if these transitions were instantaneous or gradual and what physical laws obeyed the electron during the jump.

The body of preexisting ideas, in this case supported by Schrödinger, may cause great difficulty of accepting new concepts trying to explain experimental results. Characteristically [1] remarks "as our scientific ideas develop, there will always be a tug-of-war between tradition and method: a scientist's methods may be completely empirical, yet his investigations will have no direction without the guidance of a pre-existing body of ideas...”. Such an example is the firm rejection of the concept of quantum jumps by Schrödinger as defended by Bohr. Of course, in 1926 there were neither the experimental capabilities nor the body of ideas of today. In this article Schrödinger's objections on quantum jumps are displayed and it is interesting to see how these questions have been answered today after ninety five years. Schrödinger's objections mentioned below were taken from the book "Schrödinger, Life and Thought", [2].

\section{First Objection Concerning the Deviation from Maxwell's Electromagnetic Theory}

Schrödinger: "It is claimed that the electron is in a stationary state of an atom first revolves periodically in some sort of an orbit without radiating. There is no explanation given of why it should not radiate; according to Maxwell theory it must radiate".

Schrödinger was not the only one criticizing the departure from the classical electromagnetic theory. In 1913 when Bohr's theory of atoms was discussed at a colloquium of the University and the Institute of Technology at Zurich, Max von Laue objected: "This is all nonsense! Maxwell's equations are valid under all circumstances. An electron in a circular orbit must emit radiation" [3].

This is true if one considers the electron as a very small charged particle orbiting all-round the nucleus. Electron however, is an enigmatic entity. Going through a Wilson chamber, or along the emulsion of a photographic plate, gives results compatible with a particle. On the other hand, the experiments of Davisson and Germer produced 
interference patterns characteristic of waves and the theoretical work of Louis de Broglie and David Bohm brought forth the idea of a coexistence of particles and waves which guide their motion. In quantum mechanics the electrons are described by wave functions. With this wave picture the electrons in an atom are seen not as point masses, but as standing waves analogous to vibrations in musical instruments. The specific standing waves that just "fit" around the nucleus correspond to the distinct energy levels of the Bohr's atomic model making the quantum jumps superfluous $[4,5]$.

The resolution between particle and wave according to Born has been realized by a statistical interpretation in which the square of the wave function gives the probability of finding a particle at a given position. However, if the wave is deprived of reality, it is impossible to explain the observed interference and diffraction obtained from discrete single particles. So, how to describe an electron? As Wilczek [6] remarks "an electron is a particle and a wave; it is ideally simple and unimaginably complex; it is precisely understood and utterly mysterious... No single answer does justify to reality".

\section{Second Objection Concerning the Physics of Electron Jumps}

Schrödinger: "Then the electron jumps from the orbit to another and thereby radiates. Does this transition occur gradually or suddenly? If it occurs gradually, then the electron must gradually change the rotation frequency and its energy. It is not comprehensible how this change can give sharp frequencies for spectral lines. If the transition occurs suddenly, in a jump so to speak, then indeed one can get from Einstein's formulation of light quanta the correct vibration frequency of the light, but then one must ask how the electron moves in the jump. Why doesn't it emit a continuous spectrum, as electromagnetic theory would require? And what laws determine its motion in the jump. Well, the whole idea of quantum jumps must simply be nonsense".

Schrödinger continued to deny the quantum jumps for all his life [7, 8]. A quantum jump as Bohr suggested was a sudden instantaneous event at a random moment without giving reasons why to happen that particular moment. It appeared as an event without a cause. Schrödinger and Einstein could not accept that unpredictability. Besides, Schrödinger considered meaningless to think about the behavior of single particles in real space. In 1952 [9] he wrote: "It is fair to state that we are not experimenting with single particles...We are scrutinizing records of events long after they have happened". The probability to find a system in each of its possible states given by the square of the wavefunction of Schrödinger equation is a statistical result coming from a large ensemble of this quantum system. We shall see later that this statistical character is set aside for quantum jumps by the Quantum Trajectory Theory.

As can be observed from interferometry the light from a source like a $\mathrm{Na}$ lamp consists of many individually coherent wave trains with accidental phases and planes of oscillations to each other with lengths $\mathrm{L}$ from about $0.5 \mathrm{~m}$ to $1 \mathrm{~m}$. This reveals that the jump is not instantaneous but lasts approximately from $\mathrm{t} \sim 2.10^{-9}$ to $10.10^{-9} \mathrm{sec}$, according to $\mathrm{L}=\mathrm{c} . \mathrm{t}$, where $\mathrm{c}$ is the velocity of light. Even Schrödinger had to admit that if there were jumps, the change of the atom from one energy state to another, must had a definite duration. He says accordingly..."since the wave-train which it sends out can be proved to be of considerable length...the emission must require time"... [10].

Bohr's theory of quantum jumps was impossible to be verified experimentally at that time, but in Cook and Kimble [11] proposed a method to reveal the existence of quantum jumps in an ideal isolated atom having apart from a ground state, two excited states with lifetimes differing by many orders of magnitude. This proposal was materialized in 1986, when single ions confined into electromagnetic traps and laser cooled made possible the observation of individual quantum jumps [12-14].

The experimental process goes like this: Let us suppose that the atom (in the experiment was used an ion) can be in three states with different energies, the ground state 1 , the excited state 2 , to which the transition is a "strong" one and the excited state 3 that is metastable and to which the transition is a "weak" one. The atom is simultaneously illuminated by two lasers, the one of the appropriate wavelength to excite the atom from the ground state to state 2 , the other laser suitable to excite the atom to state 3. "Strong" transition means that the probability for the atomic electron to absorb a photon when the atom is illuminated by a laser of the appropriate wavelength is high and the atomic electron returns quickly from state 2 to the ground state by radiating a photon. For the HgII ion [14] the lifetime of the state 2 is $(2.3 \pm 0.3) \mathrm{ns}$. These transitions are very rapid and the emission of photons $\left(\sim 10^{8}\right.$ photons $/ \mathrm{sec}$ ) produces a strong fluorescence. "Weak" transition means that a photon from the other laser is rarely absorbed and as state 3 is metastable with a life time about $0,1 \mathrm{~s}$, eight orders of magnitude higher than the state 2 , once excited the atomic electron stays there for a long time before relaxing to the ground state.

For most of the time the strong transition occurs and this produces a continuous bright fluorescence. For the rare occasion in which the weak transition is excited the fluorescence disappears as the electron gets stuck to the metastable state 3 and cannot be transferred between the ground state 1 and the state 2 and the atom seems dark. After a short time the electron from state 3 descends to the ground state 1, the transition between 1 and 2 prevails and the fluorescence appears again. This succession of fluorescence and darkness reveals the quantum jumps to and from the metastable state 3 in a single ion. These experimental results show that the jumps from the metastable state 3 to the ground state 1 occur suddenly and randomly in time as Bohr had proposed. However, it was impossible to detect the behavior of the electron during the jump.

\section{Experiments on an Artificial Atom}

Very interesting details of quantum jumps were revealed in 2019 by Minev, et al. [15] in Yale University in collaboration with the University of Auckland. In the experiments a macroscopic "artificial atom" was used, a superconducting circuit with quantum behavior. Like the natural atoms used in the 1986 experiments, the artificial 
atom had three discrete quantum states: a ground state, a "bright" state and a "dark" state. A beam of microwaves provided the artificial atom with energy. Absorbing or emitting photons caused jumps between the three energy states. As far as this atom is in the bright state, falling in the ground state ejects photons which can be observed as clicks from a detector. The state to and from which quantum jumps are observed is the dark one which is more stable than the bright state, so the atom would stay there for longer without emitting any photons and so appears dark.

The research team saw a succession of photons each of them signifying a decay of the bright state and the time interval between two successive photons was about a few microseconds. The sequence of photons was interrupted approximately every few hundred microseconds, apparently at random, by an interval with no photons during which the system had undergone a transition to the dark state. After a period of about 100 microseconds the flux of photons restarted from the bright state [15].

As the system was about to jump from the bright to the dark state a new phenomenon came out. Just before each jump a short interval appeared where the emission of photons decelerated, that is there was a pause warning that a jump is about to occur. As soon as the system had entered this brief pause at the end of the bright state inevitably had to fall in the dark state even without photons driving this transition. The jump into the dark state occurred randomly in time; however there was always a precursor, the brief pause, indicating the presence of a hidden cause which near the end of every bright state drives the jump inevitably to the dark state. So, the quantum jumps combine randomness in time with a deterministic character just before the jump in the dark state.

With the photons turned off and using a fine-grained time resolution the researchers found out that the jumps were gradual, continuous not sudden. This was a prediction of Quantum Trajectory Theory (QTT) a formulation of quantum mechanics which offers the possibility to describe in detail the trajectories of the individual quantum particles that obey the probabilities computed from the Schrödinger equation [16]. According to QTT quantum jumps are not instantaneous but happen in a superposition of states. Indeed, the continuous character of the jumps results from the fact that the system is in a superposition, a mixture of the two end states. By using a method called tomographic reconstruction, the researchers could figure out the relative weightings of the dark and ground states in the superposition. These weightings changed gradually over a period of a few microseconds as the jump was going between the two ends.

The electronic system monitoring the evolution of the jump was so fast that it was possible to stop or to reverse it. To "freeze" the evolution of a quantum system by rapidly repeated measurements is called Quantum Zeno effect after Zeno's arrow paradox according to which an arrow in flight is not seen to move during any single moment. This quantum effect originates from the fact that the transition probabilities between quantum states are quantratic in time. So, the probability of a transition becomes asymptotically small for very short times between repeated measurements and the quantum system collapses back onto the original state after each measurement [17, 18].

\section{Conclusion}

In 1986 experiments on natural atoms (ions) laser cooled and confined into electromagnetic traps confirmed the existence of quantum states and quantum jumps disproving the assumption that they were just artifacts, or nonsense as Schrödinger stated. Interferometry shows that the jumps are not instantaneous as the wave trains originating from them have lengths corresponding to a time interval of a few nanoseconds a fact that makes the spectral lines not absolutely sharp. In 2019 quantum jumps were studied on an "artificial atom", a superconducting circuit with three discrete quantum states. The jumps although random in time always show a preliminary phase just before them exhibiting a deterministic character. During the jumps the system is in a superposition of the initial and the final states. Very fast monitoring the evolution of the jump makes it possible to "freeze" or reverse it. These very interesting results concern the macroscopic superconducting artificial atom which can be controlled efficiently. Unfortunately, a similar control on natural atoms is impossible, so the details of quantum jumps in them are not available at least presently.

\section{References}

[1] Toulmin, S., 1957. Contemporary scientific mythology. 1st essay in metaphysical beliefs. London: SCM Press LTD.

[2] Moore, W., 1990. Schrödinger, life and thought. Cambridge: Cambridge University Press.

[3] Mehra, J. and Rechenberg, H., 1982. The historical development of quantum theory. New York: Springer Verlag. p. 1.

[4] Davies, P. C. W. and Brown, J. R., 1988. The ghost in the atom. Cambridge: Cambridge University Press.

[5] Schrödinger, E., 2010. "What is matter? ." Scientific American Nobel Prize Winning Authors, vol. 2, pp. 511.

[6] Wilczek, F., 2013. "The enigmatic electron." Nature, vol. 498, pp. 31-32.

[7] Bitbol, M., 2007. Schrödinger against particles and quantum jumps. 5th chapter in the book: Quantum mechanics at the crossroads. Springer.

[8] Schrödinger, E., 1952. "Are there quantum jumps? Part 1." The British Journal for the Philosophy of Science, vol. 3, pp. 109-123.

[9] Schrödinger, E., 1952. "Are there quantum jumps? Part 2." The British Journal for the Philosophy of Science, vol. 3, pp. 233-242.

[10] Schrödinger, E., 1935. Science and the human temperament. Chapter v. Physical science and the temper of the age. London,: George Allen and Unwin Ltd. 
[11] Cook, R. J. and Kimble, H. J., 1985. "Possibility of direct observation of quantum jumps." Physical Review Letters, vol. 54, pp. 1023-1026.

[12] Robinson, A., 1986. "Quantum jumps seen in a single ion." Nature, vol. 234, pp. 24-25.

[13] Sauter, T., Neuhauser, W., Blatt, R., and Toschek, P. E., 1986. "Observation of quantum jumps." Physical Review Letters, vol. 57, pp. 1696-1698.

[14] Bergquist, J. C., Hulet, R. G., Itano, W. M., and Wineland, D. J., 1986. "Observation of quantum jumps in a single atom." Physical Review Letters, vol. 57, pp. 1699-1702.

[15] Minev, Z. K., Mundhada, S. O., Shankar, S., Reinhold, P., Gutiérrez-Jâuregui, R., Schoelkopf, R. J., Mirrahimi, M., Carmichael, H. J., and Devoret, M. H., 2019. "To catch and reverse a quantum jump midflight." Nature, vol. 570, pp. 200-204.

[16] Ball, P., 2019. The quantum theory that peels away the mystery of measurement. Quanta Magazine.

[17] Kumar, P., Romito, A., and Snizhko, K., 2020. "Quantum zeno effect with partial measurement and noisy dynamics." Physical Review Research, vol. 2, p. 043420.

[18] Snizhko, K., Kumar, P., and Romito, A., 2020. "Quantum Zeno effect appears in stages." Physical Review Research, vol. 2, pp. 1-12. 